\title{
Images - A series of congenital mesonephric/Wolffian duct abnormalities in the adult and pediatric populations
}

Meghan Cooper ${ }^{1}$; Calvin Guiterrez ${ }^{2}$; Hubert Swana ${ }^{3}$; Mark Rich ${ }^{3}$; Lucas Wiegand ${ }^{1}$

${ }^{1}$ University of South Florida, Tampa, FL, United States; ${ }^{2}$ University of Central Florida, Orange County, FL, United States; ${ }^{3}$ Arnold Palmer Children's Hospital, Orlando, FL, United States

Cite as: Can Urol Assoc J 2020 June 5; Epub ahead of print. http://dx.doi.org/10.5489/cuaj.6344

Published online June 5, 2020

$* * *$

\section{Case report 1}

A ten-month old male developed a febrile urinary tract infection and urinary retention after hypospadias repair. Urethral catheterization was performed without difficulty. Prenatal ultrasounds had demonstrated a solitary right kidney. A repeat ultrasound revealed a solitary right kidney and a tubular pelvic structure on the left side behind the bladder. Voiding cystourethrogram (VCUG) was obtained. This revealed an irregular bladder contour and reflux into what was believed to be dilated, duplicated left ureters that ended blindly in the retroperitoneum.

The patient was managed with antibiotic prophylaxis initially and over the next several months he developed recurrent urinary tract infections and urinary retention. At 18 months of age, the patient was taken to the operating room for cystoscopy and left retrograde urography that revealed a left-sided tubular structure that coursed cranially into the retroperitoneum then downward into the pelvis. Ureterovasal fusion was demonstrated with retrograde filling of the epididymis. (Figure 1).

Robotic assisted laparoscopic excision of the left ureter was performed. Intraoperative examination of the renal fossa did not reveal a renal remnant. At two year follow up he did well with resolution of his retention and infections.

\section{Case report 2}

A two-year-old boy was referred to the pediatric urology clinic for recurrent urinary tract infections and episodes of right epididymo-orchitis. He was born with imperforate anus and underwent posterior sagittal anorectal repair at an outside institution.

Original ultrasound revealed a hydronephrotic right kidney with parenchymal thinning. Voiding cystourethrogram revealed grade 5 reflux of the right kidney and diuretic renal scanning revealed $<5 \%$ minimal function of the right kidney. Diagnostic cystoscopy and right retrograde 
were performed and demonstrated a dilated single ureter with grade 5 reflux and distal ureterovasal fusion. On the right side the ureteral orifice was cannulated, and a sensor wire passed into the right ureterovesical junction and the ureterovasal fusion as it coursed retroperitoneally through the inguinal canal to the epididymis in the right scrotum. Injection of contrast filled the vas deferens and dilated proximal ureter (Figure 2).

Patient underwent complete right nephroureterectomy and excision of distal ureterovasal fusion via a flank approach with a second lower inguinal incision. Post operatively, the patient remained infection-free. He continued to do well other than requiring clean intermittent catherization due to neurogenic bladder from a tethered cord.

\section{Discussion}

The prevalence of genitourinary abnormalities in newborns is estimated to be between 0.1-1 percent. Although these anomalies are relatively rare and often subclinical in nature, they can initially present incidentally during a workup for other pathologies. ${ }^{1}$

The establishment of the male internal reproductive system involves two key events: the formation of the testis and the maintenance and differentiation of the Wolffian (mesonephric) duct. The Wolffian duct is central in the development of the urogenital system and its role is well established. ${ }^{2}$ Embryologically, the mesonephric duct develops into the fetal kidney and bladder trigone and the distal Wolffian ducts differentiates into the urogenital structures including the epididymis, vas deferens, seminal vesicles and ejaculatory ducts. ${ }^{3}$ Although not essential for initial Wolffian duct formation, the later differentiation of this embryological structure relies on androgens derived from the Leydig cells. ${ }^{2}$

Genitourinary abnormalities due to Wolffian (mesonephric) duct malformations in pediatrics can present with symptoms of a UTI, epididymitis, during surgical exploration for undescended testes or in association with other prenatal and postnatal malformations of the genitourinary mesonephric system. ${ }^{1}$ In adults, cases often can present in a delayed fashion typically detected with the onset of sexual activity. Adults with Wolffian duct abnormalities often present with symptoms of bladder dysfunction, urinary tract infections, pelvic and ejaculatory pain or infertility. ${ }^{4}$

Ectopic ureters with ureterovasal fusion are uncommon and often associated with ipsilateral renal agenesis as well as anorectal abnormalities. ${ }^{5}$ The embryological etiology of this malformation results from the ureteral bud developing more cephalad on the mesonephric duct preventing it from achieving an independent orifice within the urinary triad. ${ }^{6}$ Therefore, the ureter remains attached to the distal Wolffian duct, which ultimately becomes the seminal vesicle and ejaculatory duct. A rudimentary renal unit is often detected on the involved side and its ureter fuses with the seminal vesicle or ejaculatory duct. ${ }^{3}$

In these images, there was noted to be ectopic ureteral insertion into the Wolffian duct structures. Each case offers a unique presentation of this genitourinary anomaly as the first 
patient had a solitary kidney with no renal remnant found, while the second case demonstrated a hydronephrotic, non-functional renal unit. However, both patients were diagnosed during a workup for recurrent urinary tract infections. This supports the concept that these anomalies are often diagnosed via ultrasound during investigation for urinary tract infection, epididymitis or flank pain in adolescent males. While in older males, the symptoms often present as prostatitis, pain on ejaculation or infertility.

\section{Conclusions}

Our two case reports demonstrate the rare clinical presentation of ureterovasal fusion in the adolescent male and offer interesting pathological anatomy. As exhibited in our cases, surgical intervention is often warranted in these patients rather than conservative therapy due to the persistence of symptoms and/or recurrence of infection. 


\section{References}

1. Shariat SF, Naderi AS, Miles B, et al. Anomalies of the wolffian duct derivatives encountered at radical prostatectomy. Rev Urol 2005;7:75-80

2. Barsoum I, Yao HH. The road to maleness: from testis to Wolffian duct. Trends Endocrinol Metab 2006;17:223-28.

3. Joseph A, Yao H, Hinton BT. Development and morphogenesis of the Wolffian/epididymal duct, more twists and turns. Dev Biol 2009;325:6-14.

4. Agarwal A, Pushkarna A, Sharma S, et al. Wolffian system agenesis with contralateral ejaculatory duct stenosis: First case in the literature. Turk J Urol 2018.

5. Matsumoto F, Suzuki M, Hosokawa S, et al. Ectopic vas deferens opening into the ureter. Int J Urol 1999;6:275-78.

6. Schwarz R, Stephens FD. The persisting mesonephric duct: high junction of vas deferens and ureter. J Urol 1978;120:592-96. 


\section{Figures and Tables}

Fig. 1. Case 1: Ureterovasal fusion was demonstrated with retrograde filling of the epididymis.

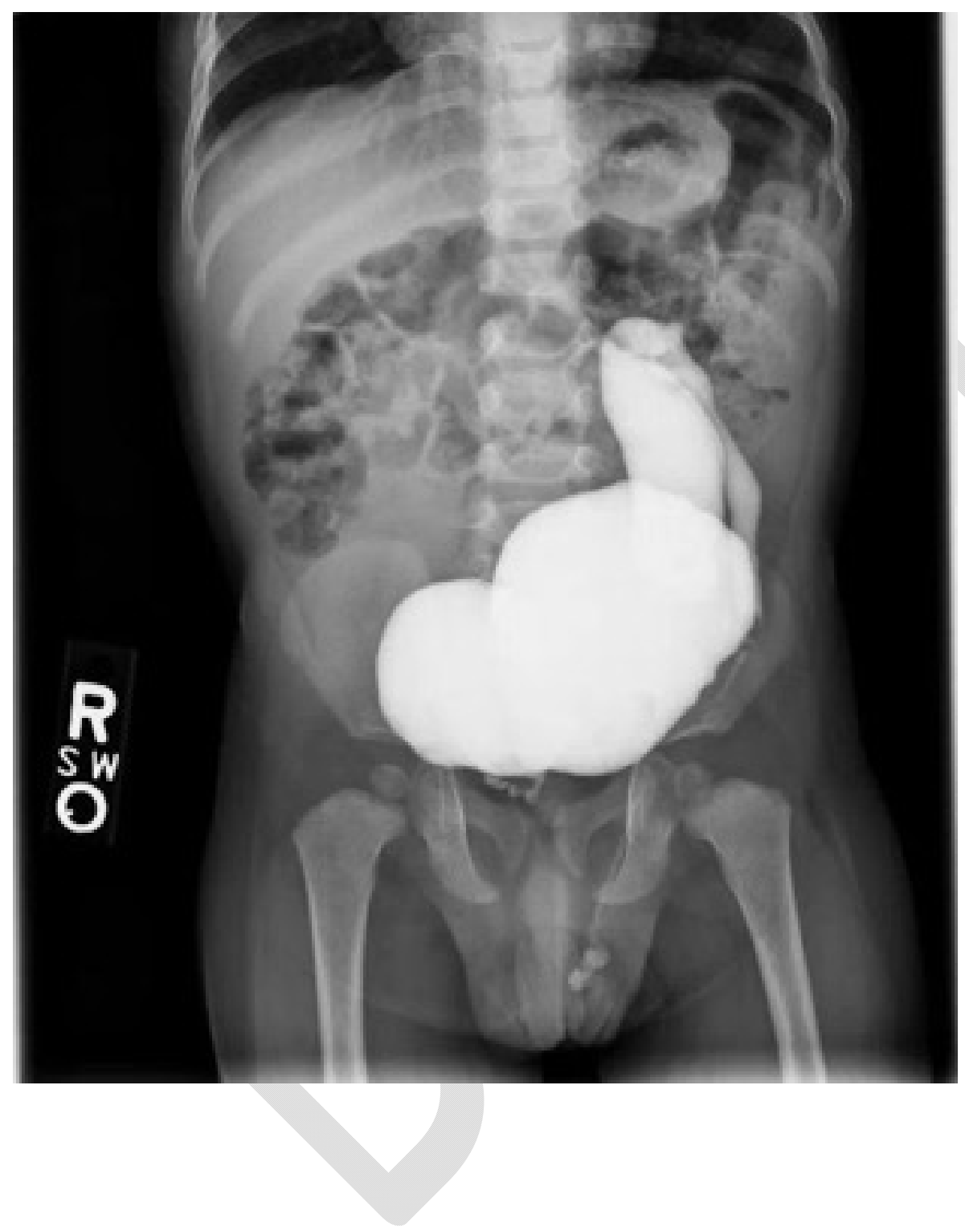


Fig. 2. Case 2: Injection of contrast filled the vas deferens and dilated proximal ureter.

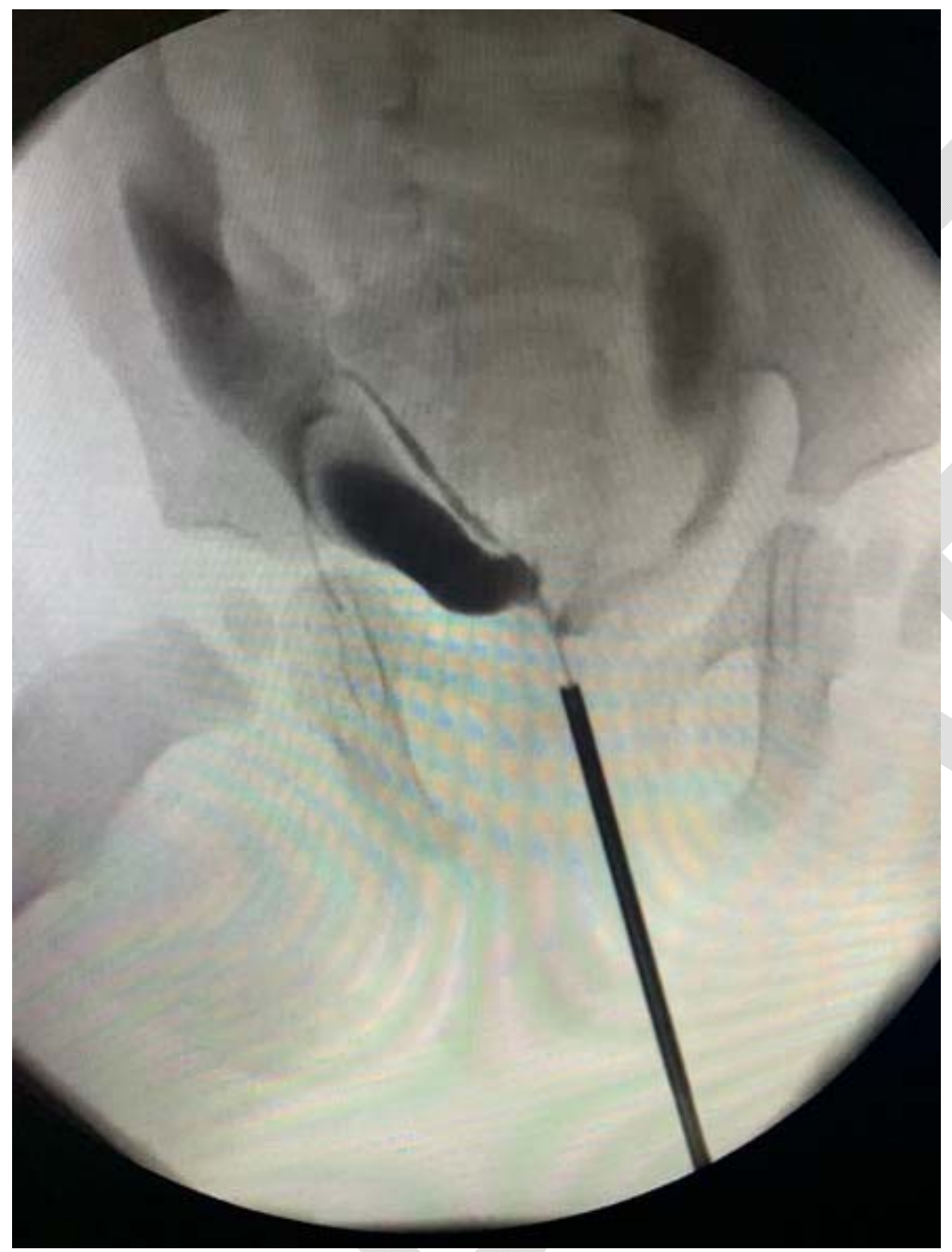

